\title{
Designing LQR Controllers for an Active Anti-roll Bar System with a Flexible Frame Model of a Single Unit Heavy Vehicle
}

\author{
Van Tan Vu*, Van Da Tran¹, Quoc Trung Pham¹, Manh Hung Truong ${ }^{1}$, Oliver Sename², \\ Peter Gaspar ${ }^{3}$

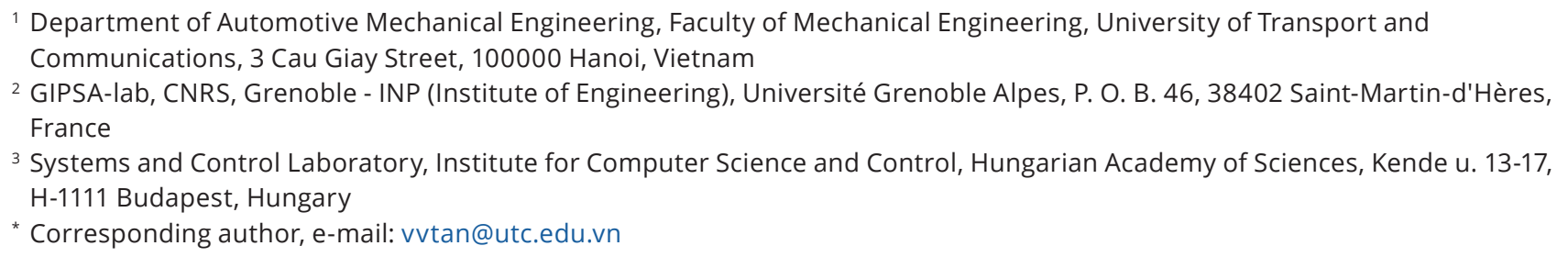

Received: 16 May 2021, Accepted: 15 June 2021, Published online: 09 August 2021

\begin{abstract}
Rollover accidents of heavy vehicles often cause serious consequences both in terms of vehicle and environmental damage as well the loss or injury of drivers, passengers and ordinary civilians. Currently, the active anti-roll bar system is considered as the most effective solution in enhancing vehicle roll stability. In this paper, we firstly investigated the role of a flexible frame of a single unit heavy vehicle in the rollover process. This approach is an important step forward in the research of the active anti-roll bar system. Then, the LQR control method is applied in designing controllers for the active anti-roll bar control system with this frame model. The active torque of the anti-roll bar system is considered as the control signal. The simulation results in the frequency and time domains with a double lane change maneuver show that the vehicle's roll stability is improved by over $30 \%$ compared to a vehicle using a passive anti-roll bar system.
\end{abstract}

Keywords

active anti-roll bar system, flexible frame, LQR control, rollover, roll stability

\section{Introduction}

The importance of heavy vehicles in terms of economic development is irrefutable and should not be underestimated, there is a necessity to conduct as much research as possible to improve the performance of such vehicles. This need is enhanced by the fact that accidents relating to these kinds of vehicles are often fatal due to the very significant mass and the large bulk (Evgenikos et al., 2016). In accordance with National Highway Traffic Safety Administration (NHTSA), In America alone there were a total of 475,000 crashes involving large trucks in 2017; resulting in 4657 fatalities, and 107,000 injuries. Apart from serious damages to other road users and adjacent population, accidents relating to heavy vehicles tend to cause severe damage to a large number of properties. Despite the fact that the year of 2017 solely recorded 21,000 out of the total crashes being due to rollover occurrence, approximately 50 percent of this figure led to death and injury (NHTSA, 2016). If roll stability of such heavy vehicles could be improved, it seems obvious that the number of fatal accidents could be decreased, and traffic safety would be increased in general.

Typically, the passive anti-roll bar is a component along with leaf or air springs and other parts constituting the suspension system on heavy vehicles. Technically, the conventional anti-roll bar is a U-shaped torsion tube that connects the right and the left elements of either solid axle or independent type of suspension system. While both tips of the anti-roll bar are mounted firmly on the axle beam, this bar is also connected to the vehicle frame at the points where it starts to bend on its right and left sides. Those elastic bushings enable the passive anti-roll bar to rotate freely at the mounting points. The two control arms should be attached as close to the wheels as possible in order to ensure up-and-down motion of the wheel rather 
than back-and-forth movement. The purpose of anti-roll bar is to provide torsional stiffness to prevent one side of the body from traveling too far in the opposite direction of the other. To be specific, given that the vehicle is turning right, due to effect of lateral acceleration, the suspension on the left-hand side is compressed and the one on the right-hand side is stretched (Miège and Cebon, 2002). The anti-roll bar at this moment will simultaneously produce two torques with opposite directions. One is to decompress the left suspension and the other is to press the right suspension, resulting in the reduction of the suspension differentiation. By doing so, the roll angle of the vehicle body is decreased, and the vehicle is kept from rolling out of corner excessively (Frimberger et al., 2000).

In general, the flexible frame contributes to the repartition of the lateral load transfer, keeping axles from generating their shares of the lateral load transfer. As a consequence, the wheels will not lift off the ground at the same time and the static roll-over threshold will be declined. Hence, the flexible frame of vehicles is really worth considering in order to provide a more accurate and thorough insight into how actual vehicles would behave in reality (Enrico, 2011).

When it comes to heavy vehicles, the most prevalent tactic to improve roll stability is an active anti-roll bar system with a pair of hydraulic actuators (Gaspar et al., 2004; $\mathrm{Vu}$ et al., 2017). As shown in Fig. 1, the active anti-roll bar system is constituted by two hydraulic actuators controlled by an electronic servo-valve, being mounted in the middle of the passive anti-roll bar on each axle. When the vehicle tilts towards one side, these actuators will produce torque to pull the wheel's opposite side, which has a tendency of lifting off, down to Earth (Vu et al., 2019).

Previous studies have mainly focused on evaluating the effectiveness of the active anti-roll bar system for a rigid model of a single unit vehicle with control methods such as neural network, PID, LQR, $\mathrm{H}_{\infty}, \mathrm{H}_{\infty} / \mathrm{LPV}$ (Boada et al., 2009; Gaspar et al., 2004; Stone and Cebon, 2010;

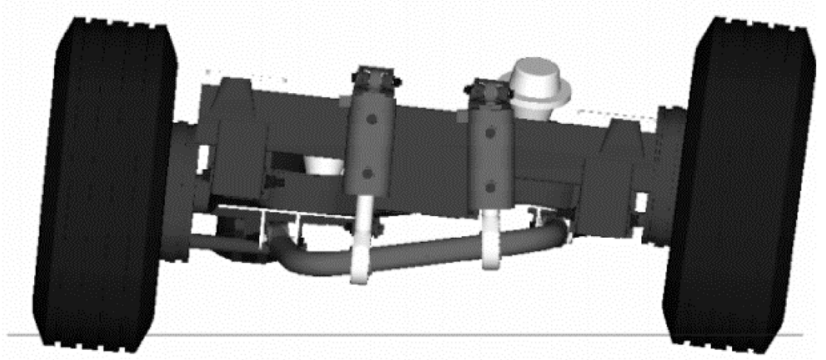

Fig. 1 Active anti-roll bar using two hydraulic actuators on heavy vehicles (Miège and Cebon, 2002)
$\mathrm{Vu}, 2017$ ). In Sampson's PhD thesis (Sampson, 2000), a proposed concept for a flexible frame model is used for the study of roll stability of heavy vehicles. However, the author had not come up with a clear solution for the application of an active anti-roll bar system.

This paper will concentrate on an active anti-roll bar system with a pair of hydraulic actuators, using torques generated by the actuators as controlled input signals on yaw-roll model with the flexible frame.

\section{Vehicle modelling}

In this section, the authors introduce the modelling of a two-axle single unit heavy vehicle with a rigid frame and a flexible frame. The symbols and parameters of both models are shown in (Gaspar et al., 2004; Vu et al., 2017).

\subsection{Yaw-roll model of linear, torsionally rigid frame single unit vehicle}

A linear rigid frame single unit heavy vehicle is modeled with three rigid components where $m_{s}$ is represented for the sprung mass and $m_{u f}$ and $m_{u r}$ are correspondingly representative of the unsprung masses at the front and rear axles including front and rear wheels and axles as shown in Fig. 2.

The dynamical equation of the model is represented in Eq. (1) (Gaspar et al., 2004; Vu, 2017):

$$
\left\{\begin{array}{l}
-m_{s, f} h_{s f} \ddot{\phi}_{f}-m_{s, r} h_{s r} \ddot{\phi}_{r}+m v(\dot{\beta}+\dot{\psi})=F_{y f}+F_{y r} \\
-I_{x z f} \ddot{\phi}_{f}+I_{z z} \ddot{\psi}-I_{x z r} \ddot{\phi}_{r}=F_{y f} l_{f}-F_{y r} l_{r} \\
\left(I_{x x f}+m_{s f} h_{s f}^{2}\right) \ddot{\phi}_{f}-I_{x z f} \ddot{\psi}=m_{s f} g h_{s f} \phi_{f}+m_{s f} v h_{s f}(\dot{\beta}+\dot{\psi}) \\
-k_{f}\left(\phi_{f}-\phi_{u f}\right)-b_{f}\left(\dot{\phi}_{f}-\dot{\phi}_{u f}\right) \\
-k_{b}\left(\phi_{f}-\phi_{r}\right)-b_{b}\left(\dot{\phi}_{f}-\dot{\phi}_{r}\right)-F_{b} h_{b}+M_{f}+T_{f} \\
\left(I_{x x r}+m_{s r} h_{s r}^{2}\right) \ddot{\phi}_{r}-I_{x z r} \ddot{\psi}=m_{s r} g h_{s r} \phi_{r}+m_{s r} v h_{s f}(\dot{\beta}+\dot{\psi}) \\
-k_{r}\left(\phi_{r}-\phi_{u r}\right)-b_{r}\left(\dot{\phi}_{r}-\dot{\phi}_{u r}\right) \\
+k_{b}\left(\phi_{f}-\phi_{r}\right)+b_{b}\left(\dot{\phi}_{f}-\dot{\phi}_{r}\right)+F_{b} h_{b}+M_{r}+T_{r} \\
-r F_{y f}=m_{u f} v\left(r-h_{u f}\right)(\dot{\beta}+\dot{\psi})+m_{u f} g h_{u f} \phi_{u f}-k_{t f} \phi_{u f} \\
+k_{f}\left(\phi-\phi_{u f}\right)+b_{f}\left(\dot{\phi}-\dot{\phi}_{u f}\right)+M_{f}+T_{f} \\
-r F_{y r}=m_{u r} v\left(r-h_{u r}\right)(\dot{\beta}+\dot{\psi})-m_{u r} g h_{u r} \phi_{u r}-k_{t r} \phi_{u r} \\
+k_{r}\left(\phi-\phi_{u r}\right)+b_{r}\left(\dot{\phi}-\dot{\phi}_{u r}\right)+M_{r}+T_{r}
\end{array} .\right.
$$

\subsection{Yaw-roll model of linear torsionally flexible single unit vehicle}

As far as the torsionally flexible frame is concerned, it means that portions of the sprung mass would roll at different angles. Thus, the chances are great that some 

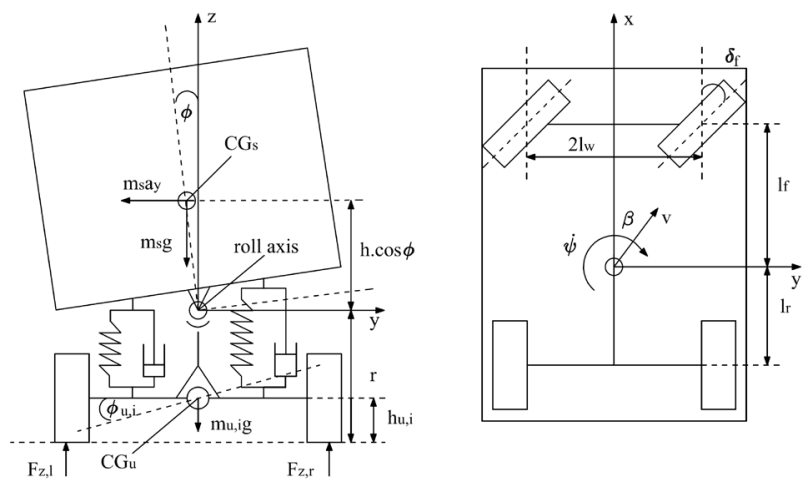

Fig. 2 Yaw-roll model of a torsionally rigid frame single unit vehicle

axles will be prevented from sharing the load transfer to counteract the overturning moment produced by the lateral acceleration. As a consequence, some wheels will be lifted sooner than others and the roll-over threshold will be reduced. Furthermore, most vehicles, if not all, in reality are manufactured with a flexible frame, meaning that analyzing the yaw-roll model of a torsionally flexible frame single unit vehicle as shown in Fig. 3 is able to provide more accuracy.

In order to capture the influence of a flexible frame, the sprung mass $\left(m_{i}\right)$ is separately split into front and rear rigid bodies $\left(m_{s f}\right.$ and $\left.m_{s r}\right)$ with their own roll angles $\left(\phi_{f}\right.$ and $\left.\phi_{r}\right)$ and appropriate inertia properties. Each possesses weight as per the following formulas (Yim et al., 2011):

$m_{s f}=\frac{m_{s} l_{r}}{l_{f}+l_{r}} ; m_{s r}=\frac{m_{s} l_{f}}{l_{f}+l_{r}}$.

Additionally, these two components of sprung mass are connected together by a torsional spring whose stiffness matches the torsional stiffness of the flexible frame. The torsional spring must be placed at the centroid height
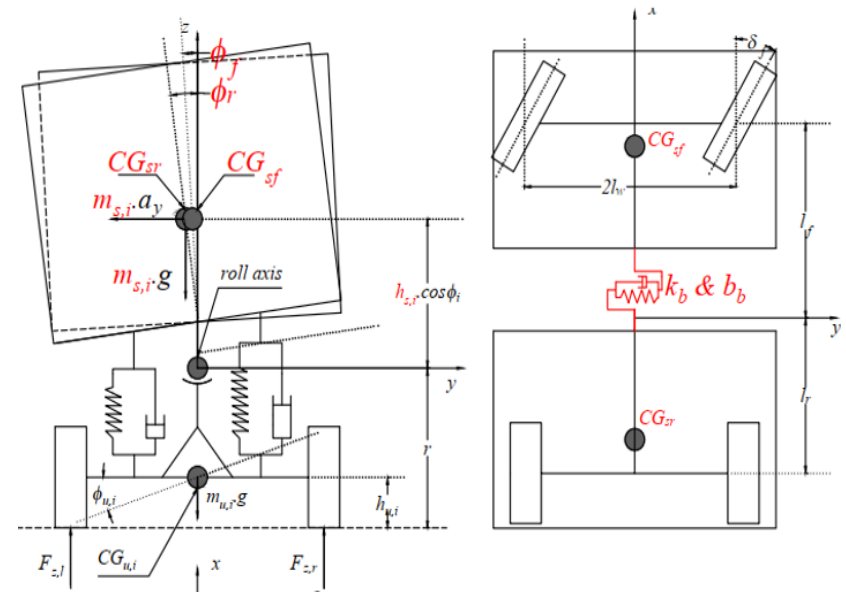

Fig. 3 Yaw-roll model of a torsionally flexible frame single unit vehicle of the frame. According to (Sampson, 2000), the torsional stiffness of the frame is about two to ten times greater than total roll stiffness of the suspension. The formula to calculate the total suspension roll stiffness is in Eq. (3):

$$
k_{\Sigma}=\frac{1}{\frac{1}{k_{f}}+\frac{1}{k_{r}}} \text {. }
$$

In this study, a frame with torsional stiffness which is 10 times higher than the total suspension roll stiffness is taken into consideration. Therefore, the frame torsional stiffness is about

$K_{b}=10 K_{\Sigma}$.

Likewise, using the formula below to find out the total suspension roll damping and the roll damping of the frame is about 10 times greater as well. However, torsional damping exerts a negligible influence on the transient response:

$b_{\Sigma}=\frac{1}{\frac{1}{b_{f}}+\frac{1}{b_{r}}} ; b_{b}=10 b_{\Sigma}$.

With a flexible frame being introduced in this section, instead of one roll angle of sprung mass like the model in Subsection 2.1, there will be two roll angles of sprung mass (one at the front axle and the other at the rear axle). This leads to an additional sprung mass roll moment equation in comparison with Eq. (1). The dynamical equation for linear torsionally flexible frame single unit vehicle is presented in Eq. (6):

$$
\left\{\begin{array}{l}
-m_{s, f} h_{s f} \ddot{\phi}_{f}-m_{s, r} h_{s r} \ddot{\phi}_{r}+m v(\dot{\beta}+\dot{\psi})=F_{y f}+F_{y r} \\
-I_{x z f} \ddot{\phi}_{f}+I_{z z} \ddot{\psi}-I_{x z r} \ddot{\phi}_{r}=F_{y f} l_{f}-F_{y r} l_{r} \\
\left(I_{x x f}+m_{s f} h_{s f}^{2}\right) \ddot{\phi}_{f}-I_{x z f} \ddot{\psi}=m_{s f} g h_{s f} \phi_{f}+m_{s f} v h_{s f}(\dot{\beta}+\dot{\psi}) \\
-k_{f}\left(\phi_{f}-\phi_{u f}\right)-b_{f}\left(\dot{\phi}_{f}-\dot{\phi}_{u f}\right)-k_{b}\left(\phi_{f}-\phi_{r}\right)-b_{b}\left(\dot{\phi}_{f}-\dot{\phi}_{r}\right) \\
-F_{b} h_{b}+M_{f}+T_{f} \\
\left(I_{x x r}+m_{s r} h_{s r}^{2}\right) \ddot{\phi}_{r}-I_{x z r} \ddot{\psi}=m_{s r} g h_{s r} \phi_{r}+m_{s r} v h_{s f}(\dot{\beta}+\dot{\psi}) \\
-k_{r}\left(\phi_{r}-\phi_{u r}\right)-b_{r}\left(\dot{\phi}_{r}-\dot{\phi}_{u r}\right)+k_{b}\left(\phi_{f}-\phi_{r}\right)+b_{b}\left(\dot{\phi}_{f}-\dot{\phi}_{r}\right) \\
+F_{b} h_{b}+M_{r}+T_{r} \\
-r F_{y f}=m_{u f} v\left(r-h_{u f}\right)(\dot{\beta}+\dot{\psi})+m_{u f} g h_{u f} \phi_{u f}-k_{t f} \phi_{u f} \\
+k_{f}\left(\phi-\phi_{u f}\right)+b_{f}\left(\dot{\phi}-\dot{\phi}_{u f}\right)+M_{f}+T_{f} \\
-r F_{y r}=m_{u r} v\left(r-h_{u r}\right)(\dot{\beta}+\dot{\psi})-m_{u r} g h_{u r} \phi_{u r}-k_{t r} \phi_{u r} \\
+k_{r}\left(\phi-\phi_{u r}\right)+b_{r}\left(\dot{\phi}-\dot{\phi}_{u r}\right)+M_{r}+T_{r}
\end{array}\right.
$$


The lateral tire force $F_{y f}$ and $F_{y r}$ at the tire-ground contacting points in the direction of the velocity are formulated in Eq. (7):

$$
\left\{\begin{array}{l}
F_{y f}=\mu C_{f} \alpha_{f} \\
F_{y r}=\mu C_{r} \alpha_{r}
\end{array}\right. \text {. }
$$

With formulas of the tire side slip angle $\alpha$ are (Vu, 2017):

$$
\left\{\begin{array}{l}
\alpha_{f}=-\beta+\delta_{f}-\frac{l_{f} \dot{\psi}}{v} \\
\alpha_{r}=-\beta+\frac{l_{r} \dot{\psi}}{v}
\end{array} .\right.
$$

Substitute Eq. (7) into Eq. (8), then we have:

$$
\left\{\begin{array}{l}
F_{y f}=-\mu C_{f} \beta+\mu C_{f} \delta_{f}-\mu C_{f} \frac{l_{f} \dot{\psi}}{v} \\
F_{y r}=-\mu C_{r} \beta+\mu C_{r} \frac{l_{r} \dot{\psi}}{v}
\end{array}\right.
$$

and

$$
\left\{\begin{array}{l}
F_{y f} l_{f}=-\mu C_{f} l_{f} \beta+\mu C_{f} l_{f} \delta_{f}-\mu C_{f} \frac{l_{f}^{2} \dot{\psi}}{v} \\
F_{y r} l_{r}=-\mu C_{r} l_{r} \beta+\mu C_{r} \frac{l_{r}^{2} \dot{\psi}}{v}
\end{array} .\right.
$$

The moments produced by the passive anti-roll bar at the front and rear axles are formulated in Eq. (10) (Vu, 2017):

$$
\left\{\begin{array}{l}
M_{r}=-4 k_{A O r} \frac{t_{a} t_{b}}{c^{2}} \phi-4 k_{A O r} \frac{t_{a}^{2}}{t_{c}^{2}} \phi_{u r} \\
M_{f}=-4 k_{A O f} \frac{t_{a} t_{b}}{c^{2}} \phi-4 k_{A O f} \frac{t_{a}^{2}}{t_{c}^{2}} \phi_{u f}
\end{array} .\right.
$$

Equation (6) is possible to be expressed in the form of the state-space representation as

$$
\left\{\begin{array}{l}
\dot{\boldsymbol{X}}=A X+B_{1} U+B_{2} W \\
\boldsymbol{Z}=C X+D_{1} U+D_{2} W
\end{array} .\right.
$$

The state vector:

$$
\begin{aligned}
\boldsymbol{X} & =\left[\begin{array}{llllllll}
\beta & \dot{\psi} & \phi_{f} & \phi_{r} & \dot{\phi}_{f} & \dot{\phi}_{r} & \phi_{u f} & \phi_{u r}
\end{array}\right] \\
\dot{\boldsymbol{X}} & =\left[\begin{array}{llllllll}
\dot{\beta} & \ddot{\psi} & \dot{\phi}_{f} & \dot{\phi}_{r} & \ddot{\phi}_{f} & \ddot{\phi}_{r} & \dot{\phi}_{u f} & \dot{\phi}_{u r}
\end{array}\right]
\end{aligned}
$$

The disturbance input: $W=\left[\delta_{f}\right]$.

The output vector: $\boldsymbol{Z}=\left[\begin{array}{llll}R_{f} & R_{r} & \phi_{f}-\phi_{u f} & \phi_{f}-\phi_{u r}\end{array}\right]$.

The mathematical relations between the normalized load transfers $\left(R_{f}\right.$ and $\left.R_{r}\right)$ and unsprung mass roll angles $\left(\phi_{u f}\right.$ and $\left.\phi_{u r}\right)$ are in Eq. (12) (Gaspar et al., 2004):

$R_{f}=\frac{k_{t f}}{l_{w} m_{f} g} \phi_{u f} ; R_{r}=\frac{k_{t r}}{l_{w} m_{r} g} \phi_{u r}$.

\section{Performance comparison between rigid and flexible} frame models

\subsection{Time response analysis}

A double lane change steering angle is employed to evaluate transitory behaviors of both rigid and flexible frame models. This double lane change maneuver is to avoid an obstacle in emergency, having a $2.5 \mathrm{~m}$ path deviation over $100 \mathrm{~m}$. The time response of the steering angle is depicted in Fig. 4. Both models are tested at the same forward velocity of $70 \mathrm{~km} / \mathrm{h}$, using only the passive anti-roll bar system.

Such performance criteria as the normalized load transfers and the suspension roll angles are going to be taken into consideration. While Fig. 5 compares the normalized load transfers at the front and rear axles, Fig. 6 shows the suspension roll angles at front and rear axles of the rigid and flexible models. Even though there are slight differences in transient responses between these two models, it is undeniable that peak values of normalized load transfers and suspension roll angles of the flexible model are higher than that of the rigid model. To be specific, the normalized load transfers of flexible vehicle are about $5.3 \%$ to $6.3 \%$ higher and the suspension roll angles are approximately $9.5 \%$ to $9.7 \%$ greater. This means that the vehicle with the flexible frame is less stable and more likely to reach its limit of suspension travel than that with the rigid frame.

Furthermore, the most obvious disparity between the rigid and flexible model is the twisting angle which is the difference between the sprung mass roll angles at both front and rear axles. When it comes to the rigid model, the sprung mass is considered as a single component, thus the twisting angle will remain zero in every situation. Whereas the sprung mass of the flexible model is divided into two bodies connected by a torsional spring and a damper. Therefore, when doing the double lane

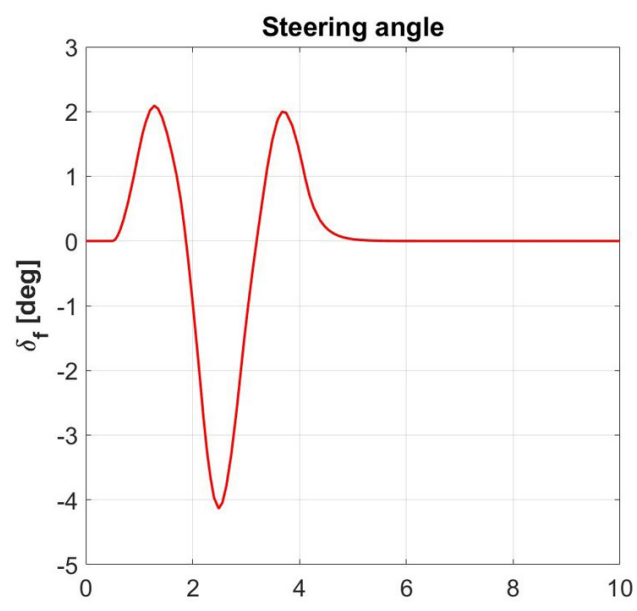

Fig. 4 Time response of the steering angle 

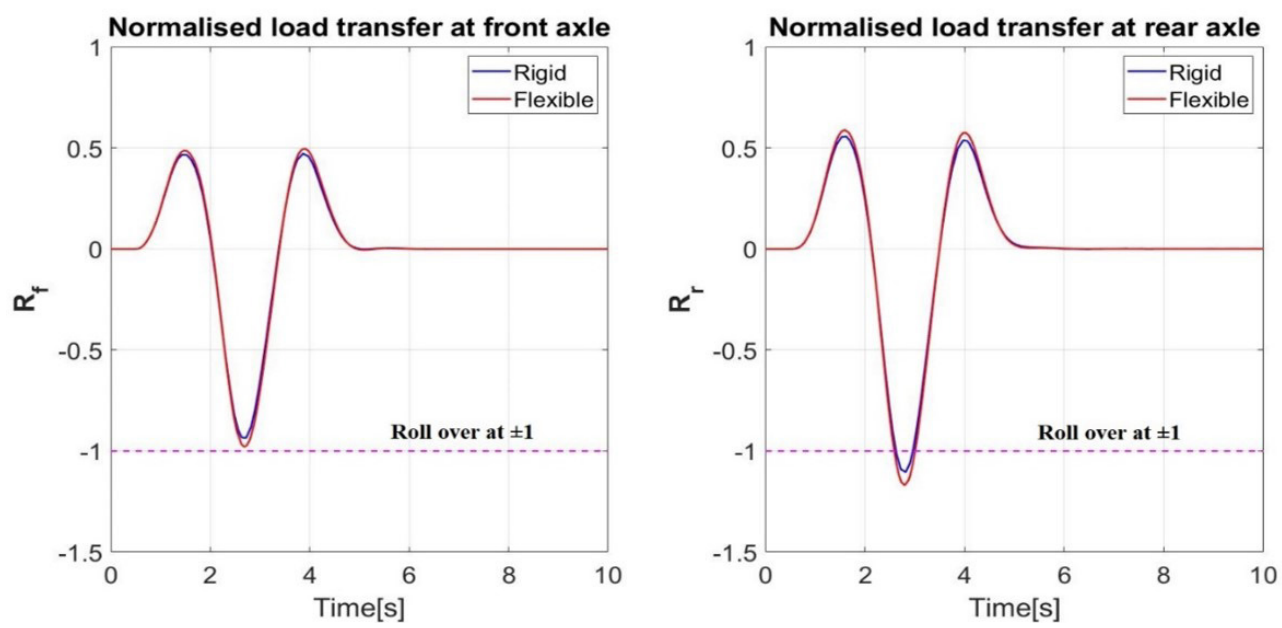

Fig. 5 Normalized load transfers of rigid and flexible frame models
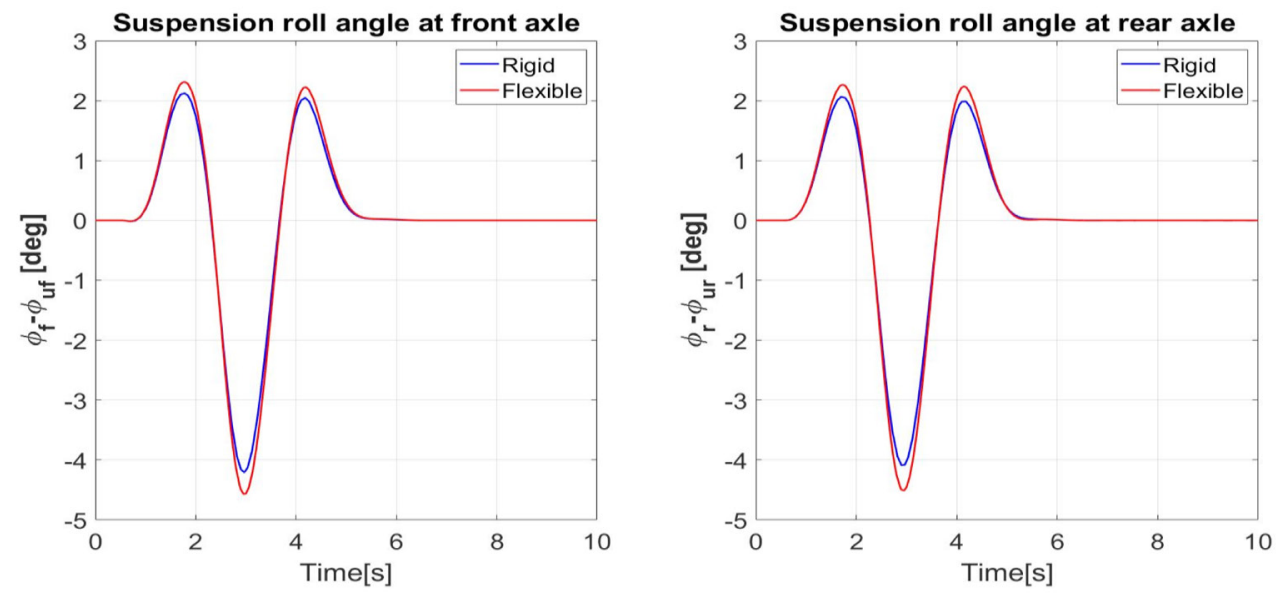

Fig. 6 Suspension roll angles of rigid and flexible frame models

change to avoid obstacle, the twisting angle of the flexible model fluctuates from around 0.03 to nearly 0.15 degrees as shown in Fig. 7. This difference between the sprung

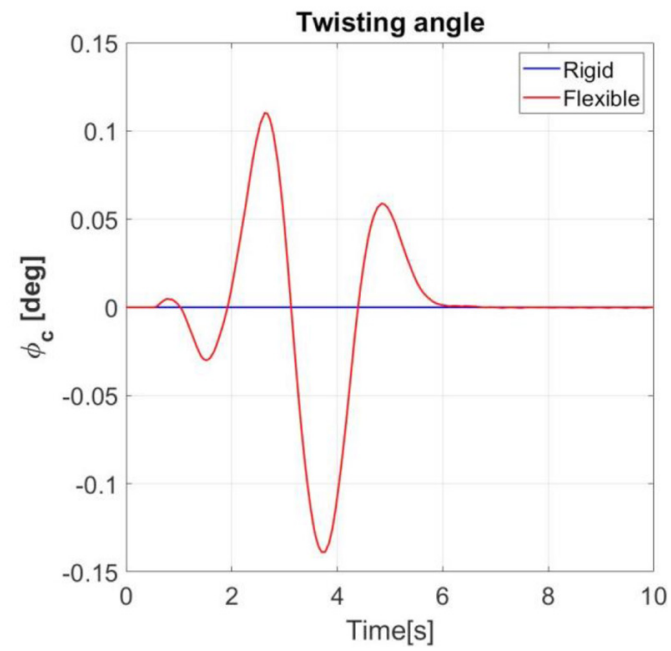

Fig. 7 Twisting angles of frames with rigid and flexible models mass roll angles at the front and rear axles plays the key element in preventing the front axle from sharing the lateral load transfer, leading to rollover threshold reduction.

\subsection{Frequency response analysis}

Frequency response functions from steering angle to the normalized load transfers and the suspension roll angles of rigid and flexible frame models are shown in Figs. 8 and 9. Similar to the previous section, both systems are also going to be tested at a forward velocity of $70 \mathrm{~km} / \mathrm{h}$. The analysis of the frequency response is to serve the purpose of studying dynamic behavior of the vehicles when subject to perturbation of the steering angle.

In fact, the frequency response can be filtered at the frequency of $4 \mathrm{rad} / \mathrm{s}$ which represents the limited bandwidth of the driver (Gaspar et al., 2004; Huang et al., 2012; Imine et al., 2014). As can be seen from Figs. 8 and 9, at low frequencies, the transfer function magnitudes of the normalized load transfers and the suspension roll angles at both 

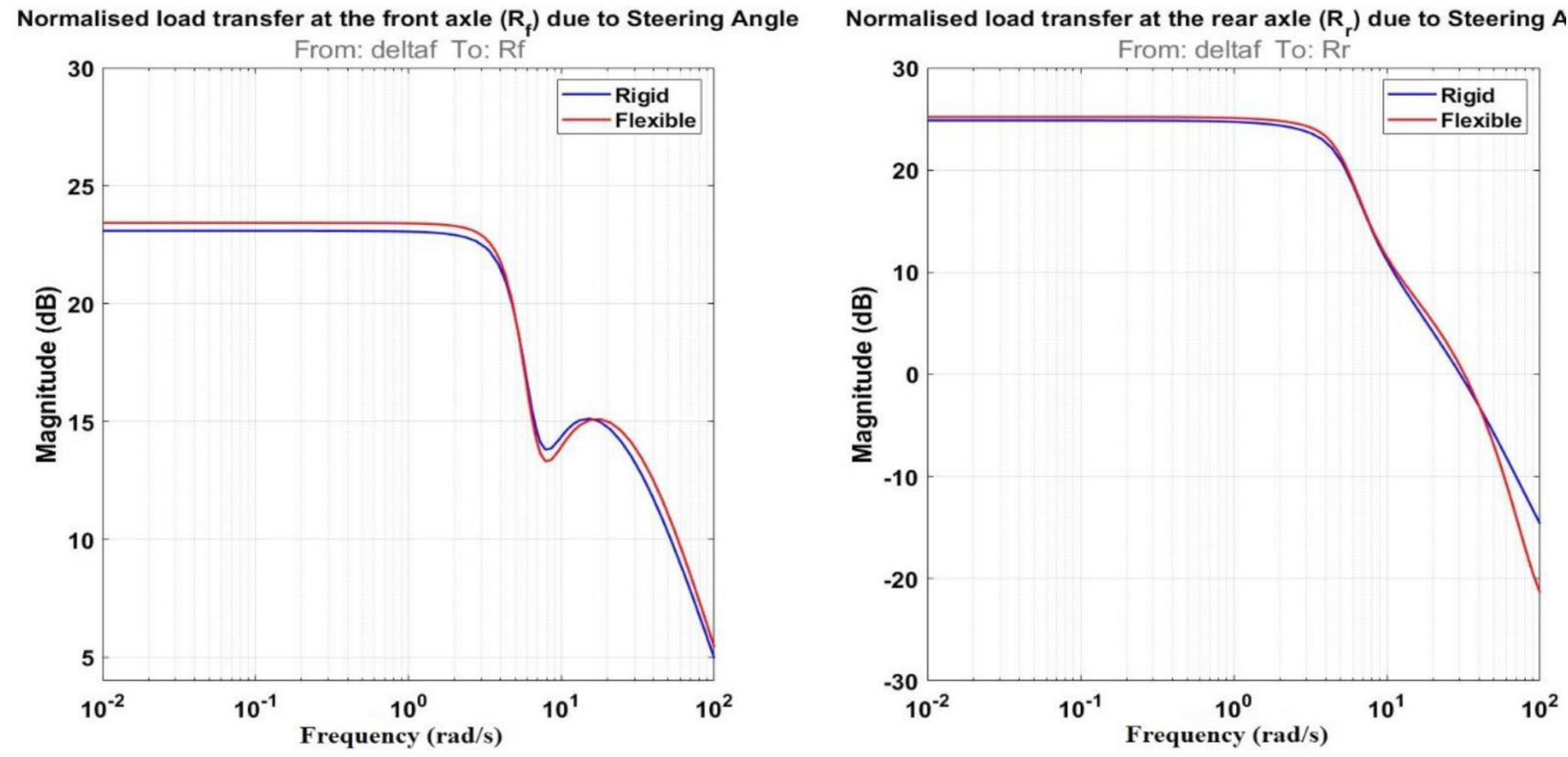

Fig. 8 Transfer function magnitude of the normalized load transfers of rigid and flexible frame models
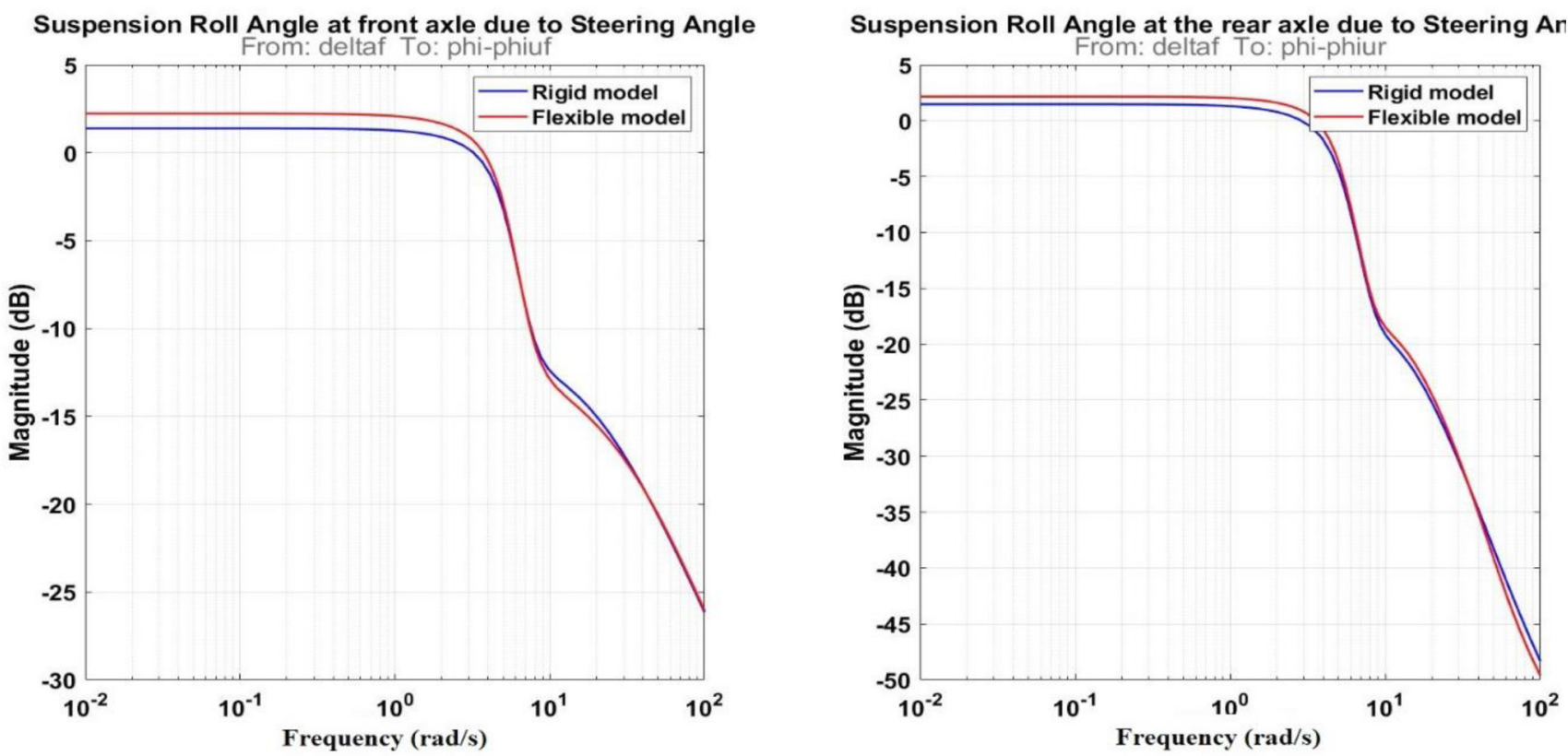

Fig. 9 Transfer function magnitude of the suspension roll angles of rigid and flexible frame models

axles of the flexible system are slightly higher than that of the rigid model. To be specific, the transfer function magnitudes of the normalized load transfers at front and rear axles of the flexible frame model are, respectively, about $0.3 \mathrm{~dB}$ and $0.4 \mathrm{~dB}$ respectively greater than that of the rigid model. Meanwhile, the differences between the flexible and rigid systems are more clear in terms of the suspension roll angle. The magnitude of the suspension roll angle transfer functions of the flexible frame are approximately $0.84 \mathrm{~dB}$ and $0.68 \mathrm{~dB}$ higher than the figure of the rigid model at both front and rear axles correspondingly. Hence, the normalized load transfers and the suspension roll angles of vehicles with a flexible frame are more sensitive to the steering angle in comparison with the rigid model.

\section{Designing LQR active anti-roll bar controllers}

\subsection{Linear Quadratic Regulator control background}

The linear approach basically starts with the conversion of a non-linear system into a linear. Thus, the Linear TimeInvariant (LTI) dynamical modeling is commonly adopted 
for control and observation purposes for both SingleInput-Single-Output (SISO) system and Multi-InputMulti-Output (MIMO) system. The Linear Time-Invariant dynamic system equations are given by

$$
\left\{\begin{array}{l}
\dot{x}(t)=A x(t)+B_{01} u(t)+B_{02} w(t) \\
z(t)=C x(t)+D_{01} u(t)+D_{02} w(t)
\end{array},\right.
$$

where $x(t)$ denotes state, $w(t)$ is disturbance, $u(t)$ represents control input and $z(t)$ is the output vectors.

A general LQR controller as shown in Fig. 10 is using the feedback signal from output $z(t)$ to generate appropriate control input $u(t)$. For the LQR controller design, it assumes that all states of the system are able to be measured or estimated. The main objective of an LQR controller is technically to determine the control input $u$ in order to minimize performance index $J$. This index consists of not only performance characteristic requirements but also the controller input limitations, which is usually presented by ( $\mathrm{Vu}, 2017)$ :

$J=\int_{0}^{\infty}\left(x^{T} Q x+u^{T} R u+2 x^{T} N u\right) \mathrm{d} t$

where $Q, R$ and $N$ are positive definite weighting matrices.

Consider the state feedback control law as:

$u=-K x$,

where $K$ denotes the state feedback gain matrix and has the following form $K=R^{-1} B^{T} P$ and where the matrix $P$ is the solution of Riccati equation as follows:

$A P+A^{T} P-P B R^{-1} B^{T} P+Q=0$

The optimal closed-loop system is achieved from Eqs. (13) and (15) as

$\dot{x}(t)=\left(A-B_{01} K\right) x(t)+B_{02} w(t)$.

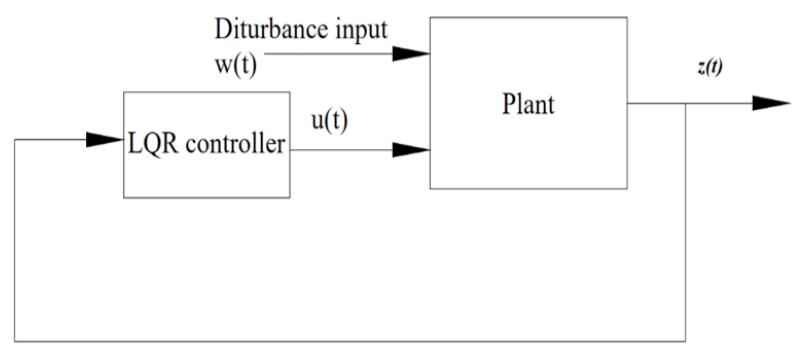

Fig. 10 LQR controller diagram
In next section, the solution to find $K$ and choosing LQR weighting matrices $Q, R, N$ will be presented in detail.

\subsection{LQR controller design}

The main objective of designing an LQR controller for an active anti-roll bar system is to minimize rollover occurrence of vehicles. Because the normalized load transfers $\left(R_{f, r}\right)$ and the suspension roll angles $\left(\phi_{f, r}-\phi_{u f, u r}\right)$ have direct impacts on the vehicle roll stability, these characteristics must be decreased to meet control objectives. Furthermore, the torques generated by the active anti-roll bar system at front and rear axles are required to be within the bearable saturation of the actuators. Therefore, the performance index $J$ in Eq. (14) is chosen as follows:

$J=\int_{0}^{\infty}\left(\begin{array}{l}\rho_{1} R_{f}^{2}+\rho_{2} R_{r}^{2}+\rho_{3}\left(\phi_{f}-\phi_{u f}\right)^{2}+\rho_{4}\left(\phi_{r}-\phi_{u r}\right)^{2} \\ +R_{t f} T_{f}^{2}+R_{t r} T_{r}^{2}\end{array}\right) \mathrm{d} t$,

where $\rho_{1}, \rho_{2}, \rho_{3}, \rho_{4}, R_{t f}, R_{t r}$ are weighting parameters of the performance index $J$.

The selection of parameters in Eq. (17) permits a larger set of different criteria concentrating on the active antiroll bar performance and/or the restriction of actuators' saturation.

In order to evaluate how well the active anti-roll bar performs, there will be two distinct LQR controllers designed and put into analogy:

- The first controller design (LQR1): This controller aims at penalizing the normalized load transfers at both axles and loosening the limitation of the actuators. The weighting parameters' values are chosen as $R_{t f}=R_{t r}=10^{-5} ; \rho_{1}=\rho_{2}=2.10^{4} ; \rho_{3}=\rho_{4}=1$.

- The second controller design (LQR2): Unlike LQR1, in this case the LQR2 targets decreasing the suspension roll angles. But it also relaxes the actuators' saturation. Therefore, the set of weighting parameters is:

$$
R_{t f}=R_{t r}=10^{-5} ; \rho_{1}=\rho_{2}=1 ; \rho_{3}=\rho_{4}=2.10^{6} \text {. }
$$

The matrices $Q, R, N$ of the performance index $J$ are determined as follows: 
$Q=\left[\begin{array}{cccccccc}0 & 0 & 0 & 0 & 0 & 0 & 0 & 0 \\ 0 & 0 & 0 & 0 & 0 & 0 & 0 & 0 \\ 0 & 0 & \rho_{3} & 0 & 0 & 0 & -\rho_{3} & 0 \\ 0 & 0 & 0 & \rho_{4} & 0 & 0 & 0 & -\rho_{4} \\ 0 & 0 & 0 & 0 & 0 & 0 & 0 & 0 \\ 0 & 0 & 0 & 0 & 0 & 0 & 0 & 0 \\ 0 & 0 & -\rho_{3} & 0 & 0 & 0 & \rho_{1}\left(\frac{k_{t f}}{l_{w} \times m_{f} \times g}\right)^{2}+\rho_{3} & 0 \\ 0 & 0 & 0 & -\rho_{4} & 0 & 0 & 0 & \rho_{2}\left(\frac{k_{t r}}{l_{w} \times m_{r} \times g}\right)^{2}+\rho_{4}\end{array}\right]$

$R=\left[\begin{array}{cc}R_{t f} & 0 \\ 0 & R_{t r}\end{array}\right] ; N=\operatorname{zeros}(8,2)$.

\section{Simulation results analysis}

\subsection{Response to a double lane change steering input}

A double lane change maneuver is a popular test for heavy vehicles because it is often used to avoid obstacles in an emergency. The time response of the steering angle is depicted in Fig. 4. The vehicle is also evaluated at the forward velocity of $70 \mathrm{~km} / \mathrm{h}$. The responses of the linear torsionally flexible frame single unit model using passive and two LQR active anti-roll bar controllers to double lane change steering angle are depicted in Figs. 11, 12.

The normalized load transfers at the rear axle in this case builds up faster than at the front axle due to the increase of the ratio of suspension stiffness to the vertical load at the rear axle. With the passive system, the inner wheel at the

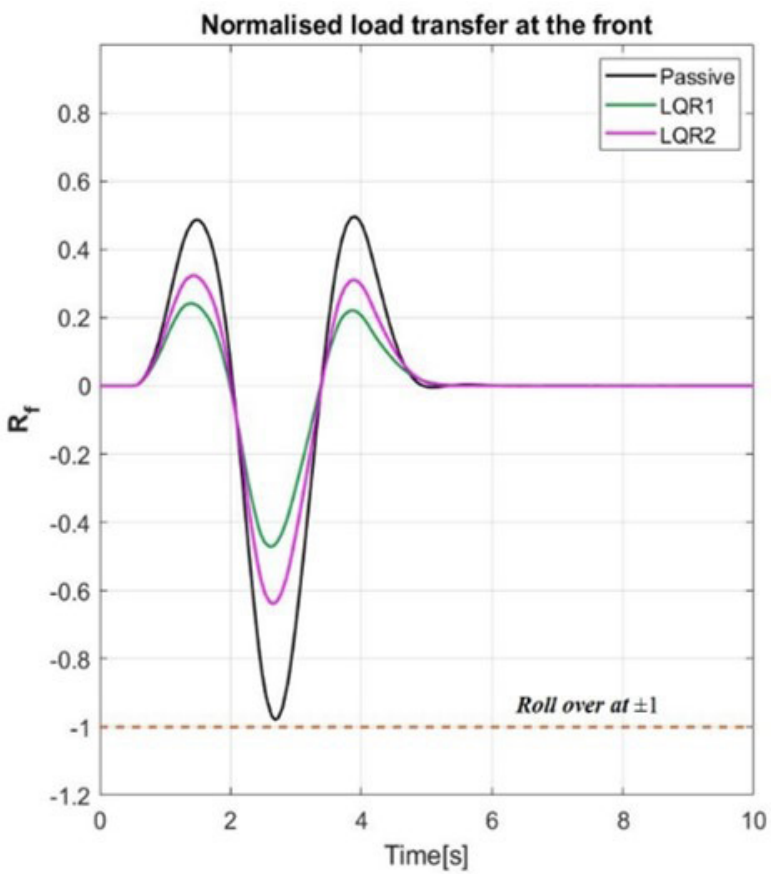

rear axle lifts off ground because the normalized load transfer $R_{r}$ exceeds -1 , while the inner wheel at the front axle still remains in contact with the ground. Conversely, both active anti-roll bar systems manage to pull down the peak values of the normalized load transfers at both axles so that the rollover incidents are prevented. The comparisons measured in percentage among load transfers of two active and passive systems are presented in Table 1.

When it comes to the suspension roll angles, the responses of all three systems stay under limit of the suspension travel. Table 2 shows analogies in the suspension roll angles among passive, LQR1 and LQR2 active antiroll bar systems in proportion.

It is also necessary to re-check the quality of the active systems by ensuring the torques generated do not exceed the saturation of the actuators of $120 \mathrm{kN}$ ( Vu, 2017). Obviously, as shown in Fig. 13, the active anti-roll bar system using

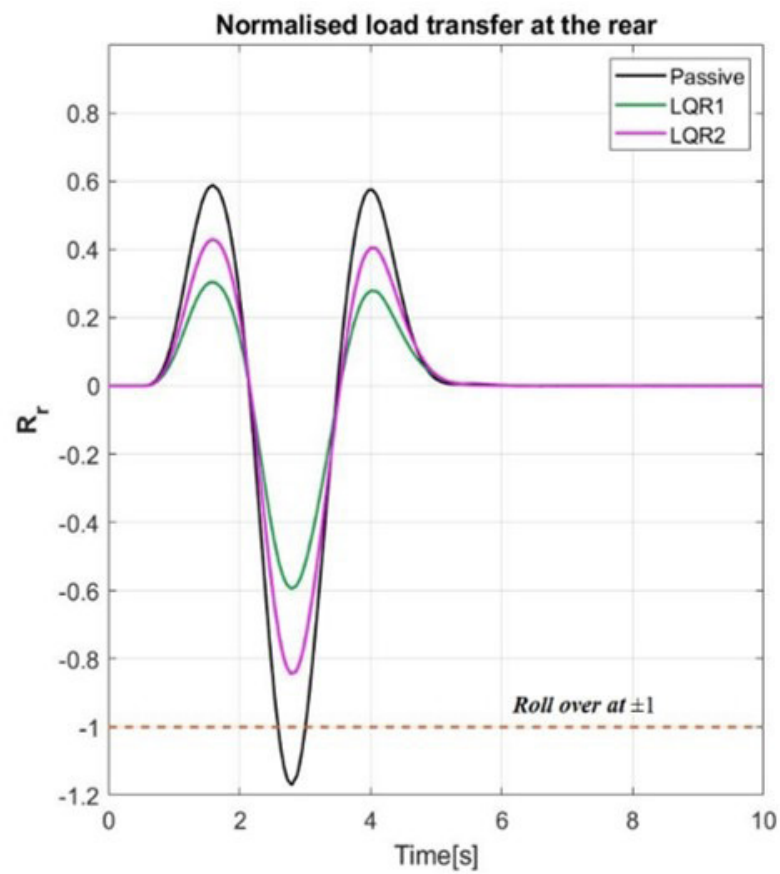

Fig. 11 Time response of normalized load transfers to double lane change steering input 

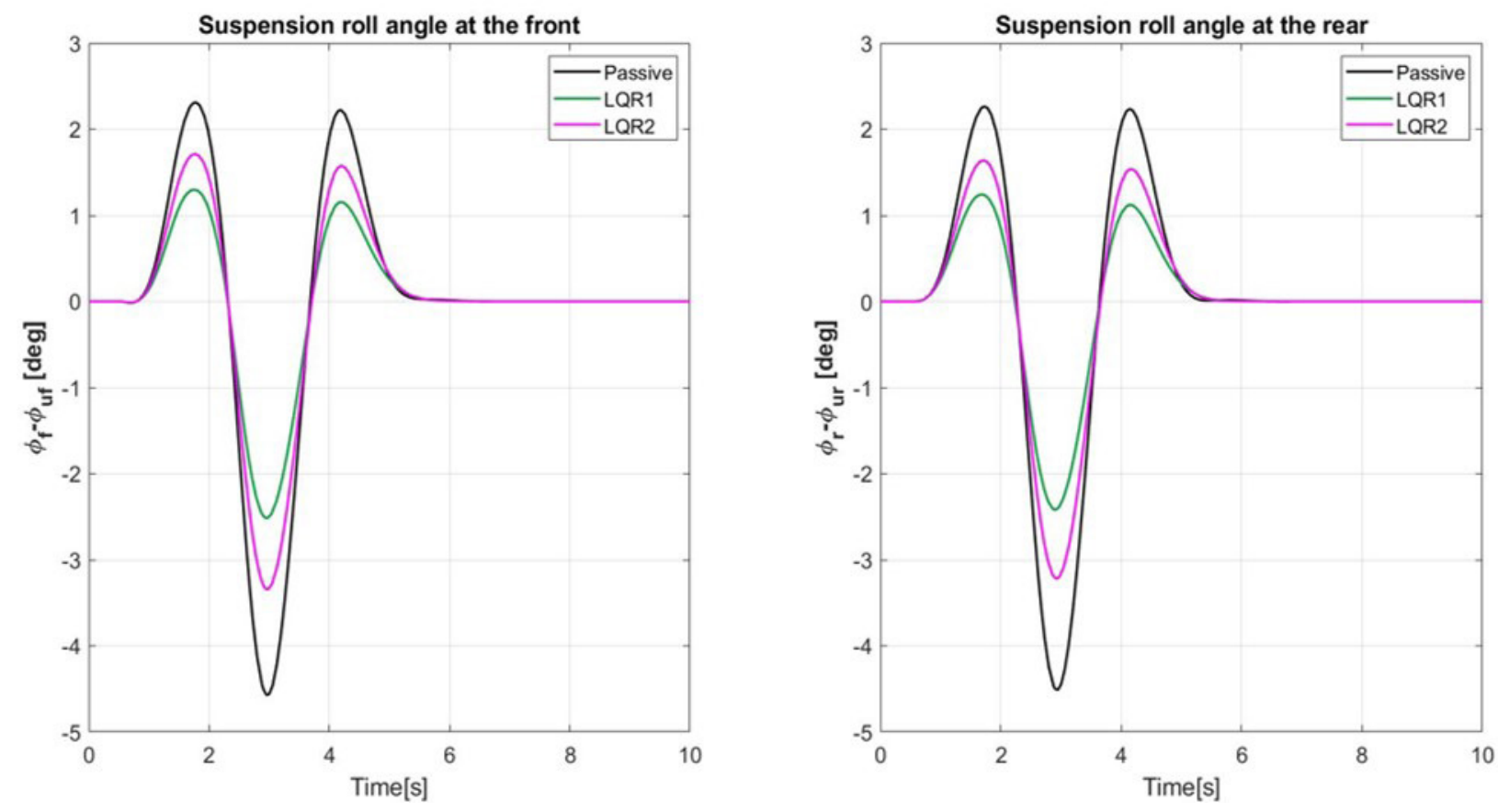

Fig. 12 Time response of suspension roll angles to double lane change steering input

Table 1 Comparisons of peak value of normalized load transfers in cases of passive, LQR1 and LQR2 active systems

\begin{tabular}{lccc}
\hline $\begin{array}{l}\text { Normalized } \\
\text { load transfer }\end{array}$ & Passive & LQR1 & LQR2 \\
\hline$R_{f}$ & $100 \%$ & $48 \%$ & $65 \%$ \\
$R_{r}$ & $100 \%$ & $50 \%$ & $72 \%$ \\
\hline
\end{tabular}

Table 2 Comparisons of peak value of suspension roll angles in cases of passive, LQR1 and LQR2 active systems

\begin{tabular}{lccc}
\hline $\begin{array}{l}\text { Suspension } \\
\text { roll angle }\end{array}$ & Passive & LQR1 & LQR2 \\
\hline$\phi_{f}-\phi_{u f}$ & $100 \%$ & $55 \%$ & $73 \%$ \\
$\phi_{r}-\phi_{u r}$ & $100 \%$ & $53 \%$ & $71 \%$ \\
\hline
\end{tabular}

LQR1 controller produces about $23 \mathrm{kN}$ and $29 \mathrm{kN}$ of torques at the front and rear axles respectively. In the meantime, the peak values of torque that were generated by the LQR2 controller are roughly $21 \mathrm{kN}$ at the front and $22 \mathrm{kN}$ at the rear.

\subsection{Frequency response analysis}

The analysis of frequency response is essential to study the dynamic behavior of the vehicles when subject to perturbation of the steering angle. The frequency response was filtered at the frequency of $4 \mathrm{rad} / \mathrm{s}$ and the systems were tested at a speed of $70 \mathrm{~km} / \mathrm{h}$.

As shown in Fig. 14, with the active anti-roll bar system using LQR1 controller, the transfer function magnitude of the normalized load transfer at the front axle is decreased down to around $18 \mathrm{~dB}$. At the other axle, the magnitude of load transfer function falls to $20 \mathrm{~dB}$.
However, the LQR2 active controller is only able to reduce the magnitude of normalized load transfer by $3 \mathrm{~dB}$ at the front and by $2 \mathrm{~dB}$ at the rear axles. Table 3 gives detailed comparison about the transfer function magnitude of the normalized load transfers in all three cases.

It is also noticeable that the normalized load transfer response of the passive system starts rolling off at $4 \mathrm{rad} / \mathrm{s}$ while that of both active controllers begins to roll off sooner at $2 \mathrm{rad} / \mathrm{s}$.

\section{Conclusions}

In this study we investigated the flexible frame model of a single unit heavy vehicle. The simulation results in the time and frequency domains showed that the level of roll instability of the vehicle with a flexible frame is more sensitive than considering the rigid frame, which is consistent with the reality of the rollover phenomena for heavy vehicles. LQR control method is applied on the active anti-roll bar system for the flexible frame models. The active torque of the active anti-roll bar system is considered as the control signal. With the simulation results in the frequency and time domains with a double lane change, the vehicle roll stability is improved by over $30 \%$ compared to the same vehicles using the passive anti-roll bar system.

The characteristic coefficients for the flexible frame have a great influence on the properties of the control system, so the study of these coefficients is the next potential research direction. 

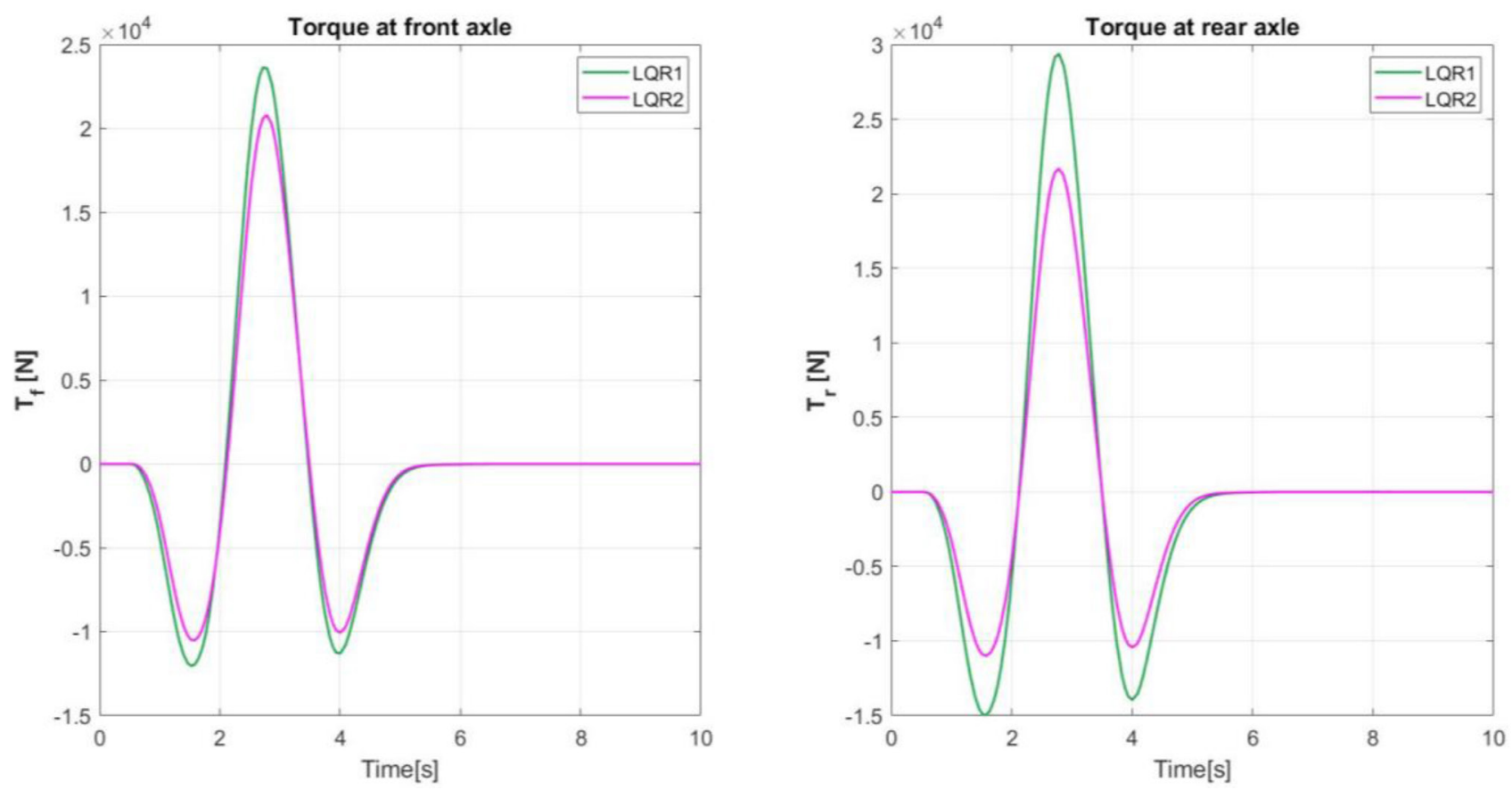

Fig. 13 Torques generated by active anti-roll bar system in response to double-lane change steering input
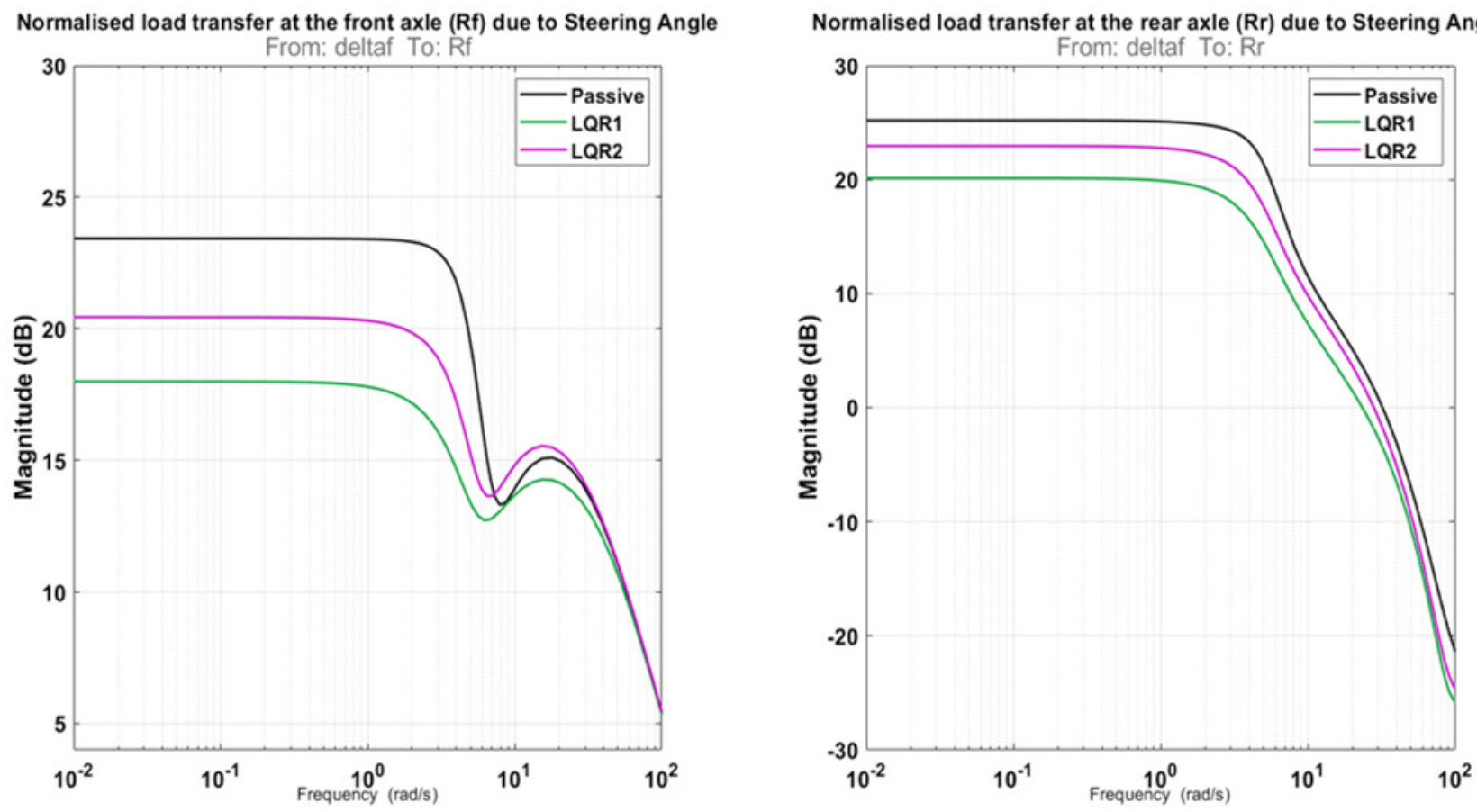

Fig. 14 Transfer functions of normalized load transfers when using passive, LQR1 and LQR2 active anti-roll bar systems

Table 3 Transfer function magnitude of normalized load transfers in cases of passive, LQR1 and LQR2 active systems

\begin{tabular}{lccc}
\hline Transfer function & Passive & LQR1 & LQR2 \\
\hline$\frac{R_{f}}{\delta_{f}}$ & $23.4 \mathrm{~dB}$ & $18 \mathrm{~dB}$ & $20.4 \mathrm{~dB}$ \\
$\frac{R_{r}}{\delta_{r}}$ & $25.2 \mathrm{~dB}$ & $20 \mathrm{~dB}$ & $22.9 \mathrm{~dB}$ \\
\hline
\end{tabular}

\section{Acknowledgment}

This work has been supported by the University of Transport and Communications through the key project T2019-CK-012TD. 


\section{References}

Boada, M. J. L., Babe, A. G., Boada, B. L., Calvo Ramos, J. A., Lopez, V. D. (2009) "Active roll control using reinforcement learning for a single unit heavy vehicle", International Journal of Heavy Vehicle Systems, 16(4), pp. 412-430. https://doi.org/10.1504/IJHVS.2009.027413

Enrico, S. (2011) "Modelling chassis flexibility in vehicle dynamics simulation", PhD Thesis, University of Surrey.

Evgenikos, P., Yannis, G., Folla, K., Bauer, R., Machata, K., Brandstaetter, C. (2016) "Characteristics and Causes of Heavy Goods Vehicles and Buses Accidents in Europe", Transportation Research Procedia, 14, pp. 2158-2167. https://doi.org/10.1016/j.trpro.2016.05.231

Frimberger, M., Wolf, F., Scholpp, G., Schmidt, J. (2000) "Influences of Parameters at Vehicle Rollover", In: International Bod Engineering Conference, Detroit, MI, USA, Paper number: 2000-01-2669. https://doi.org/10.4271/2000-01-2669

Gaspar, P., Szaszi, I., Bokor, J. (2004) "The Design of a Combined Control Structure to Prevent the Rollover of Heavy Vehicles", European Journal of Control, 10(2), pp. 148-162.

https://doi.org/10.3166/ejc.10.148-162

Huang, H-H., Yedavalli, R. K., Guenther, D. A. (2012) "Active roll control for rollover prevention of heavy articulated vehicles with multiple-rollover-index minimisation", Vehicle System Dynamics, 50(3), pp. 471-493.

https://doi.org/10.1080/00423114.2011.597863

Imine, H., Benallegue, A., Madani, T., Srairi, S. (2014) "Rollover Risk Prediction of Heavy Vehicle Using High-Order Sliding-Mode Observer: Experimental Results", IEEE Transactions on Vehicular Technology, 63(6), pp. 2533-2543.

https://doi.org/10.1109/TVT.2013.2292998
Miège, A., Cebon, D. (2002) "Design and Implementation of an Active Roll Control System for Heavy Vehicles", In: $6^{\text {th }}$ International Symposium on Advanced Vehicle Control, AVEC 2002, Hiroshima, Japan, pp. 10-21.

NHTSA (National Highway Traffic Safety Administration) (2016) "Traffic Safety Facts Annual Report Tables", [online] Available at: https://cdan.nhtsa.gov/tsftables/tsfar.htm [Accessed: 05 July 2020]

Sampson, D. (2000) "Active Roll Control of Articulated Heavy Vehicles", $\mathrm{PhD}$ Thesis, University of Cambridge.

Stone, E. J., Cebon, D. (2010) "Control of semi-active anti-roll systems on heavy vehicles", Vehicle System Dynamics, 48(10), pp. 1215-1243. https://doi.org/10.1080/00423110903427439

Vu, V. T. (2017) "Enhancing the roll stability of heavy vehicles by using an active anti-roll bar system", PhD Thesis, University of Grenoble Alpes.

Vu, V. T., Sename, O., Dugard, L., Gaspar, P. (2017) "Enhancing roll stability of heavy vehicle by LQR active anti-roll bar control using electronic servo-valve hydraulic actuators", Vehicle System Dynamics, 55(9), pp. 1405-1429. https://doi.org/10.1080/00423114.2017.1317822

Vu, V. T., Sename, O., Dugard, L., Gaspar, P. (2019) "An Investigation into the Oil Leakage Effect Inside the Electronic Servo-valve for an $\mathrm{H}_{\infty}$ /LPV Active Anti-roll Bar System", International Journal of Control, Automation and Systems, 17(11), pp. 2917-2928. https://doi.org/10.1007/s12555-019-0060-2

Yim, S-Y., Yoon, J-Y., Cho, W-K., Yi, K-S. (2011) "An Investigation on Rollover Prevention Systems: Unified Chassis Control versus Electronic Stability Control with Active Anti-Roll Bar", Proceedings of the Institution of Mechanical Engineers, Part D: Journal of Automobile Engineering, 225(1), pp. 1-14. https://doi.org/10.1243/09544070JAUTO1444 